\title{
恒河猴骨髓间充质干细胞在体外向角膜 上皮前体细胞的诱导分化
}

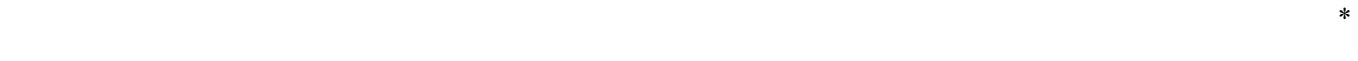 \\ (1) 中山大学中山眼科中心, 眼科学国家重点实验室, 广州 510060; (2) 中山大学干细胞和组织工程中心, 广州 510080; \\ (3) 中山大学附属第三医院普外科, 广州 510630.*联系人, E-mail: gejian@mail.sysu.edu.cn)
}

\begin{abstract}
摘要 组织工程生物角膜是角膜的理想替代物, 为严重眼表疾病的治疗带来了希望. 然而, 足量种子细 胞的获得是构建生物角膜的瓶颈. 骨髓间充质干细胞(MSC)具有多向分化潜能. 为探讨 MSC 能否分化 为角膜上皮细胞, 将分离培养的灵长类动物恒河猴 P3 代 MSC 在体外基质微环境中通过 3 种方案诱导, 对诱导前后的 MSC 用形态学观察、免疫组织化学技术和流式细胞仪检测评价其分化情况. 结果显示, III组 MSC 表现出角膜上皮前体细胞的形态特征，检测出角膜上皮前体细胞的标志，包括 integrin $\beta 1$, Cx43, Pax6 和 P63 的表达. 这说明, 在适宜条件下, MSC 能够诱导分化为角膜上皮前体样细胞, 有可能 作为构建生物角膜的种子细胞来源, 进行眼表修复.
\end{abstract}

关键词 恒河猴 骨髓间充质干细胞 角膜上皮细胞 去上皮羊膜基质 Transwell 体系 细胞生长因子 脂多糖

角膜上皮细胞(corneal epithelial cell, EC)的稳定 对维持角膜的透明性至关重要. 其中, 角膜上皮再生 的来源是角膜缘干细胞, 干细胞缺乏常常严重损伤 眼表, 造成视力下降, 甚至致盲. 对此类疾病, 传统 的异体板层角膜移植供体来源受限. 采用自体角膜

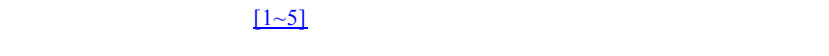
${ }^{[6-9]}$ 对部分患者有良好疗效, 但无法适用于双眼角膜 病患者. 采用唇黏膜移植重建眼表虽有报道 ${ }^{[10]}$, 但 临床效果较差. 近几年, 组织工程生物角膜为解决眼 表重建难题带来了希望. 生物角膜的核心问题是获 得具有生物活性的种子细胞. 胚胎干细胞具有分化 全能性 ${ }^{[11 ~ 17]}$, 是替代治疗的理想种子细胞, 但因其 存在伦理争议及致瘤性等问题而受到严重制约.

最近，采用骨髓间充质干细胞 (mesenchyaml stem cell, MSC)移植治疗眼表损伤在动物实验中取得 了良好效果 ${ }^{[18-20]}$. MSC存在多潜能性, 在合适的条件 下可诱导细胞向特定方向分化. 与胚胎干细胞相比, 这类细胞取材方便, 易于体外分离培养, 避免了伦理 争议, 且可来自自体组织, 避免了免疫排斥反应, 具 有更广阔的应用前景. 因此, 本研究探索了 MSC向
$\mathrm{EC}$ 分化的可能性, 目的在于为构建生物角膜提供有 效的、来源丰富的种子细胞.

\section{1 材料与方法}

（i）实验动物. 选用 2 岁龄健康雄性恒河猴, 由中山眼科中心实验动物中心提供, 经检疫符合实 验动物标准. 本研究获得中山大学道德伦理委员会 批准.

(ii) $\mathrm{MSC}$ 的分离及培养 ${ }^{[21]}$. 无菌条件下用含肝 素(6000 U)的注射器抽取骨髓 $5 \sim 15 \mathrm{~mL}$, 将细胞悬液 按 $1: 1$ 比例加入到Percoll $(1.073 \mathrm{~g} / \mathrm{mL})$ 密度梯度离 心(2000 r/min, $25 \mathrm{~min})$, 离心后小心吸取富含有核细 胞的界面层, 洗涤 1 2 次, 离心, 去上清, 用 $1 \mathrm{~mL}$ 培 养液重悬细胞. 将此混悬液接种于 $25 \mathrm{~cm}^{2}$ 培养瓶中 $37^{\circ} \mathrm{C}, 5 \% \mathrm{CO}_{2}$ 培养箱中培养, 每 $3 \mathrm{~d}$ 换液 1 次. 培养 液为L-DMEM (Gibco), 内含 $10 \%$ FBS (Gibco)、100 $\mathrm{U} / \mathrm{mL}$ 青霉素、100 mg/mL链霉素、2.4 mg/mL HEPES 和 $3.7 \mathrm{mg} / \mathrm{mL} \mathrm{NaHCO}$. 原代细胞达 $80 \%$ \% $0 \%$ 以上融 合时进行传代. 传代细胞经成骨、成脂诱导实验检测 其多向分化潜能 ${ }^{[22]}$. 
(iii) MSC 的体外标记. 用苂光染料 cM-DiI (Leiden, the Netherlands)标记 MSC ${ }^{[23,24]}$. 标记前用 PBS清洗细胞, 将 $3 \mu \mathrm{g} / \mathrm{mL}$ 的cM-DiI加入培养细胞, $5 \% \mathrm{CO}_{2}$ 培养箱中 $37^{\circ} \mathrm{C}$ 孵育 $5 \mathrm{~min}$, 再置入 $4^{\circ} \mathrm{C}$ 孵育 15 $\mathrm{min}$, 弃去苂光染液, 洗涤 3 次后, 加入新鲜培养基于 培养箱内培养, 次日在共聚焦显微镜(Zeiss, Germany) 下观察细胞标记情况.

(iv) EC原代培养. 无菌条件下分离恒河猴角膜 板层, 将角膜缘上皮面朝上平铺于培养血中, 加入 Dispase $(1.2 \mathrm{U} / \mathrm{mL})$ 消化 $2 \mathrm{~h}$ 分离角膜上皮层与角膜基 质, 然后加入 $0.1 \%$ 胰蛋白酶和 $0.01 \%$ EDTA消化上皮 层 $15 \mathrm{~min}$, 终止消化后收集细胞离心, 弃上清, 用培 养液重悬细胞, 计数, 按 $5 \times 10^{3}$ 个 $/ \mathrm{cm}^{2}$ 密度接种到 Transwell6 孔培养系统的插入子中, 加入角膜上皮细 胞专用培养液 ${ }^{[25]}$ 放置于 $100 \mathrm{~mm}$ 培养血中培养, 每日 观察细胞生长情况.

（v）去细胞羊膜基质. 无菌状态下取保存的羊 膜组织 ${ }^{[26,27]}$. 使用前, 将羊膜浸泡在含抗生素的PBS 中, 清洗后剪成比 6 孔培养板孔径稍大的圆形, 上皮 面朝上贴在培养血底壁，加入 $0.02 \%$ EDTA, $37^{\circ} \mathrm{C}$ 条 件下消化 $2 \mathrm{~h}$, 然后用细胞刮小心刮除上皮层, 用 PBS洗净刮下的上皮碎片. 将去上皮羊膜平铺在 Transwell6 孔培养板底部, 过夜待稍干燥后加入少许 培养基备用.

(vi) MSC 向 EC 的诱导. (1) 共孵育体系的制 备. 将标记 MSC 接种至预置有去上皮羊膜的 6 孔板 中, 加入基础培养基培养，当细胞达 $80 \%$ 融合时，与 Transwell6 孔培养系统的插入子(含 $80 \%$ 融合的 EC) 汇合, 进行 MSC-EC 非接触共培养(图 1). 按汇合时 记为 $0 \mathrm{~d}$, 分 3 组进行实验： I 组加入基础培养液, 由 $90 \%$ L-DMEM 和 $10 \% \mathrm{FBS}$ 组成; II 组加入条件培养 液, 由基础培养液添加 $10 \mu \mathrm{g} / \mathrm{mL}$ 胰岛素、 $8 \mu \mathrm{g} / \mathrm{mL}$ 维 甲酸、 $10 \mathrm{ng} / \mathrm{mL}$ 表皮细胞生长因子和 $10 \mathrm{ng} / \mathrm{mL}$ 碱性 成纤维细胞生长因子(Sigma)组成; III 组加入条件培 养基, 由 II 组条件培养液添加 $1 \mu \mathrm{g} / \mathrm{mL}$ 脂多糖 (LPS, Sigma)组成. 培养液每隔 $1 \mathrm{~d}$ 换液 1 次. $10 \mathrm{~d}$ 后收集标 本检测. (2) 形态学观察. $10 \mathrm{~d}$ 后, 相差显微镜下观察 细胞的形态变化. 进行电子显微镜检测前, 将组织块 取出放置平血中, 用 PBS 漂洗 3 次, 切成 $3 \times 1 \times 1$ 的小 条加入 4\%戊二醛 $1 \mathrm{~mL}$ 固定, PBS 清洗后梯度酒精脱 水. 进进行扫描电子显微镜检测时, 将组织放入醋酸 异戊酯中 $15 \mathrm{~min}$, 临界点干燥 $3 / \mathrm{h}$ 左右后金属镀膜,
在扫描电子显微镜下观察; 进行透射电子显微镜检 测时, 组织用环氧树脂Epon812 包埋, 常规超薄切片, 醋酸双氧铀及枸椽酸铅双染色, 透射电子显微镜下 观察. (3) 免疫苂光检测. $10 \mathrm{~d}$ 后, 取各组组织标本进 行冰冻切片, 采用兔抗 Integrin $\beta 1, \mathrm{Cx} 43$ (Boster)和鼠 抗 Pax6, P63 (Chemicon)单克隆抗体免疫组织化学鉴 定. 组织切片用纯丙酮固定 $10 \mathrm{~min}$, PBS 清洗, $0.2 \%$ Triton X-100 孵育 $10 \mathrm{~min}, 2 \%$ 牛血清白蛋白阻断非特 异性染色, PBS 清洗后分别滴加 4 种抗体 $4^{\circ} \mathrm{C}$ 过夜, PBS 清洗, FITC 苂光标记二抗 $37^{\circ} \mathrm{C}$ 孵育 $45 \mathrm{~min}, \mathrm{PBS}$ 清洗, 苂光抗淬灭剂封片, $4^{\circ} \mathrm{C}$ 避光保存, 共焦显微镜 下观察. 阴性对照用非免疫血清代替一抗. (4) 流式 细胞检测. $10 \mathrm{~d}$ 后, 消化离心收集细胞, PBS 清洗后制 成细胞悬液, 破膜. 破膜后的细胞分别加入单抗 Integrin $\beta 1, C x 43$, Pax6 和 P63, 避光孵育 $30 \mathrm{~min}$, 实 验管洗涤后, 分别加入相应 IgG-FITC 二抗各 $100 \mu \mathrm{L}$, 避光孵育 $30 \mathrm{~min}$. 各管洗涤后, 各加入 PBS $300 \mu \mathrm{L}$, 流式细胞仪检测, Cell-Quest 软件分析计算阳性细胞 数. 阴性对照采用 IgG-FITC 二抗单独孵育.

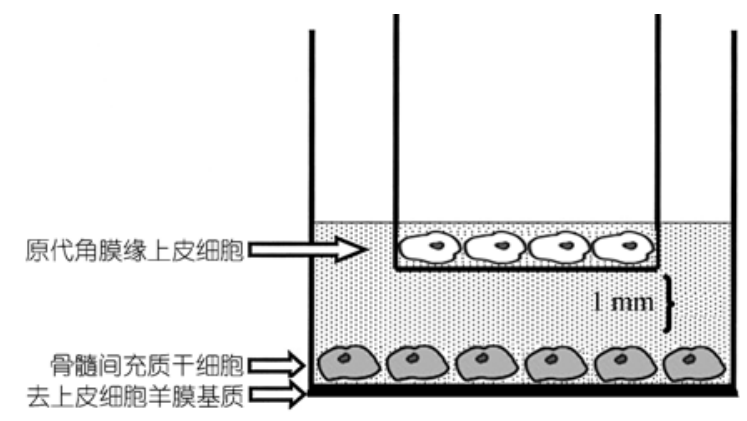

图 1 Transwell 共孵育体系示意图 $\mathrm{EC}$ 培养至插入子内, $c \mathrm{M}-\mathrm{DiI}$ 标记的 MSC 培养至预置有羊膜的 6 孔板内

(vii) 统计学方法. 实验至少重复 3 次, 结果采 用 SPSS13.0 软件分析. 计量资料均采用 $\bar{x} \pm s$ 表示, 检验水准 $\alpha=0.05$.

\section{2 结果}

\subsection{MSC 培养}

原代MSC接种的最初数日, 培养液中悬浮的血 细胞成分随时间延长逐渐崩解, 并随换液次数而被 清除. $3 \mathrm{~d}$ 后可见红细胞中间有少量贴壁的单个散在 梭形细胞. $6 \mathrm{~d}$ 后细胞数目增多, 成集落式生长. 10 13 $\mathrm{d}$, 细胞长满瓶底. 经传代后, 细胞生长较快, 细胞呈 长梭形, 有突起, $4 \sim 7$ d 即可汇合成单层, 原代和传代 
MSC形态及生长特点无明显改变. 传代细胞经成骨、 成脂诱导后能成功分化为骨细胞和脂肪细 胞, 证实 细胞具有多向分化潜能(图 2).

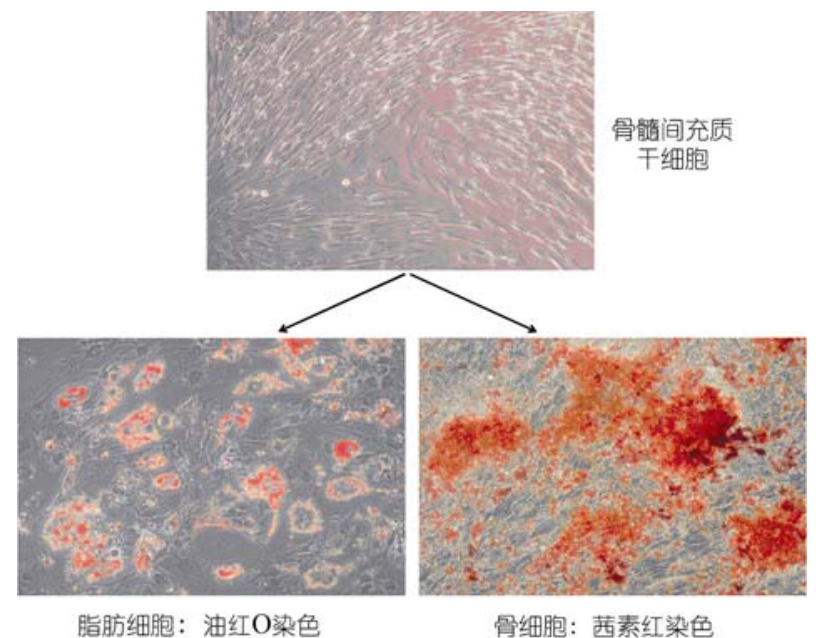

图 $2 \mathrm{MSC}$ 经成骨、成脂诱导实验证实有多向分化潜能

用cM-DiI标记MSC后共焦显微镜下可见胞膜呈 红色苂光, 形态保持良好. 标记早期, 细胞形态呈菼 光环状, 培养数天后, 细胞中苂光颗粒增多, 苂光增 强, 细胞核未染苂光. 其阳性率达到 98\%以上(图 3).

\subsection{MSC 向 EC 的诱导分化}

( i ) 形态学检测. 相差显微镜下可见去上皮羊 膜表面平滑. 诱导 $10 \mathrm{~d}$ 后, | 组与 || 组羊膜表面的 $\mathrm{MSC}$ 呈典型长梭形, 与诱导前细胞无明显变化; III 组 MSC可见部分细胞呈多角形 (图 4). 扫描电子显微镜 下可见去上皮羊膜基质表面为丝状胶原纤维 (图 5(a)). 诱导 $10 \mathrm{~d}$ 后, I 组和 II 组可见丝状胶原纤维上的细胞 为细长梭形, 细胞表面有短而粗的微线毛突起, 梭形 细胞胞浆向两极伸出长突起相互连接 (图 5 (b)和(c)); III 组细胞呈多角形, 排列紧密, 紧贴于其 下的纤维状基质 (图 5(d)). 透射电子显微镜下可见去 上皮羊膜基质板层纤维排列规则，相邻板层间纤维 走向相互垂直 (图 5(e)). 诱导 $10 \mathrm{~d}$ 后, I 组和 II 组细 胞呈梭形, 胞核较大, 染色质分布稀疏, 电子致密度 较低, 胞浆内线粒体、高尔基器、内质网等细胞器发 达, 这种特征符合典型MSC的超微结构特点(图 5(f) 和 $(\mathrm{g}))$; III 组细胞呈多角形, 细胞间伸出突起相互连 接, 细胞间还可见到类似上皮细胞之间特征性的胞 膜局部电子密度增高的缝隙连接(图 5(h)).

( ii ) 免疫菼光检测. 诱导前 MSC 表达 Integrin $\beta 1$, 不表达 Cx43, Pax6 和 P63 (图 6). 诱导 10 $\mathrm{d}$ 后, Integrin $\beta 1$ 在 3 组细胞中均有阳性表达, 以 I 组 最强; Cx43, Pax6 和 P63 在 I 组细胞中均未检测到明 显表达; Cx43 和 Pax6 在 II 组细胞中有微弱表达, 在 III 组表达较明显; P63 在 II 组细胞中未检测到明显表 达, 在 III 组有弱表达(图 7).

(iii) 流式检测. 诱导 $10 \mathrm{~d}$ 后, II 和 II组细胞中 Integrin $\beta 1$ 阳性细胞百分率较 I 组显著下降; II 和 III 组细胞中 Cx43 和 Pax6 阳性细胞百分率则较 I 组显 著增加; II 组细胞中 P63 阳性细胞百分率较 I 组无显 著差异, 而 III 组细胞中 P63 阳性细胞百分率较 I 和 II 组显著增加(图 8). 这说明, III 组更适合诱导 MSC 向 EC 分化; II 组虽有一定诱导作用, 但效果不明显; I 组则无明显诱导效果.

\section{3 讨论}

$\mathrm{MSC}$ 是一类存在于骨髓的非造血类的原始胚胎 样细胞群, 在合适的条件下可诱导细胞向特定方向 分化. 早期的研究已证实, MSC可以向骨细胞、软骨 细胞、脂肪细胞等多种中胚层细胞分化 ${ }^{[22,28]}$. 跨胚层

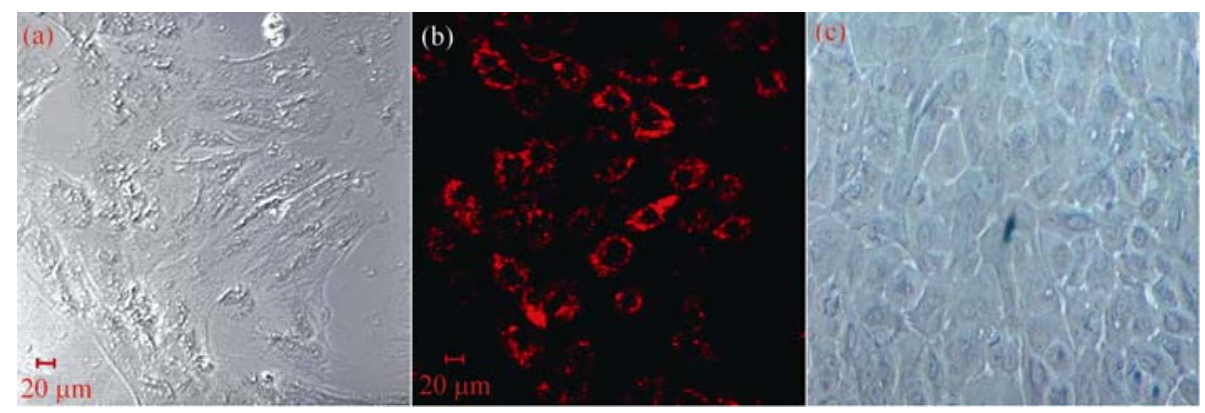

图 3 Transwell 共孵育体系中的细胞

(a)和(b) MSC 经 cM-DiI 标记后, 胞膜呈红色菼光; (c) 相差显微镜下观察原代EC呈多角形或卵圆形 $(\times 200)$ 

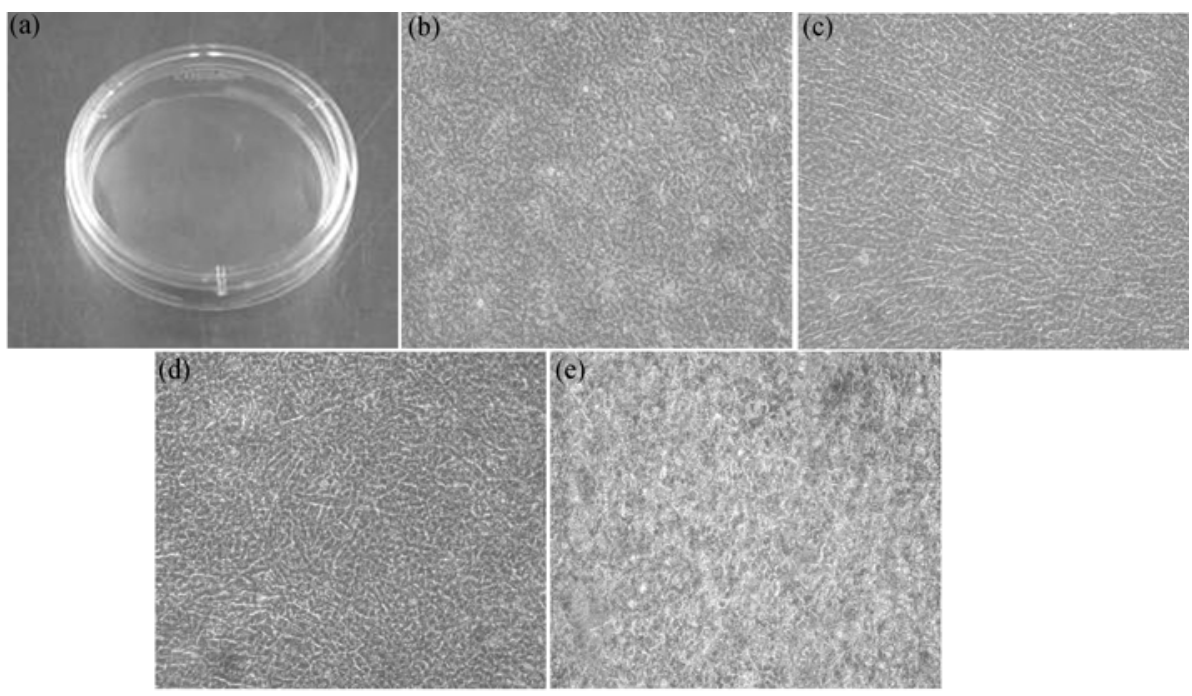

图 4 去上皮羊膜的肉眼形态 $(\mathrm{a})$ 和光学显微镜下形态 $((\mathrm{b}), \times 200)$

MSCs 与 EC 共孵育培养 $10 \mathrm{~d}$ 后 I 组(c)、II 组(d)和 III组 $(e)$ 细胞在光子显微镜下的形态 $(\times 100)$

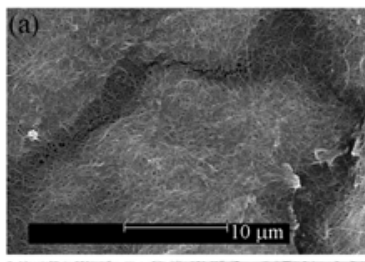

(e)

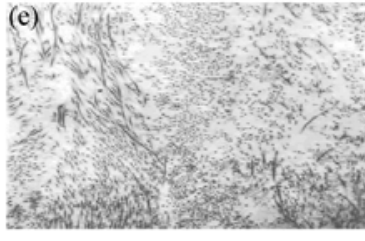

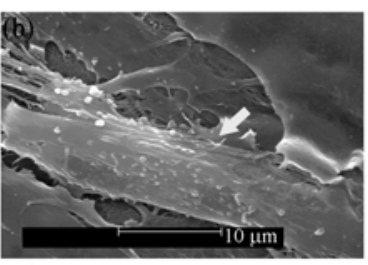

$-2$

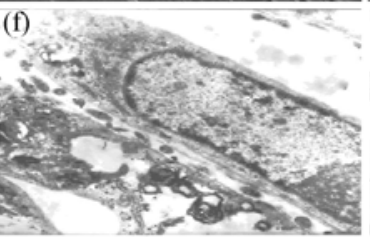

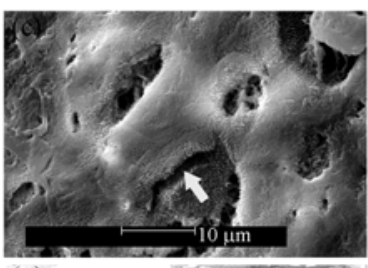

(g)

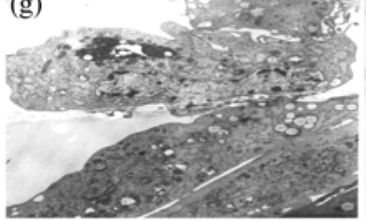

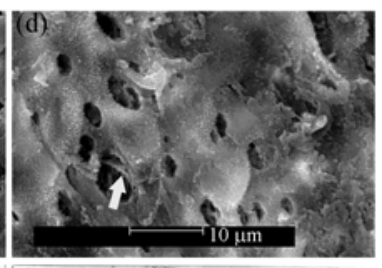

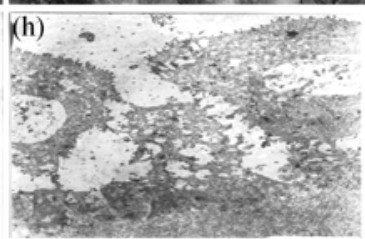

图 5

扫描电子显微镜下可见去上皮羊膜基质表面由丝状胶原纤维组成(a); 诱导 $10 \mathrm{~d}$ 后，１组(b)和 II 组(c)可见羊膜表面的细胞(箭头所示)呈长梭形; III组(d)细胞(箭头所示)呈多角形; 透射电子显微镜下可见羊膜基质板层规则排列的胶原纤维(e); 诱导 $10 \mathrm{~d}$ 后, I 组(f)和 II 组 $(\mathrm{g})$ 细胞呈梭性, 胞 核较大, 胞浆内细胞器丰富; 111 组 (h) 细胞呈多角形, 与基质连接紧密, 细胞间可见胞膜局部电子密度增高的缝隙连接. 放大倍数：(a)和(b), $\times 7000 ;(\mathrm{c}),(\mathrm{d}),(\mathrm{g})$ 和 $(\mathrm{h}), \times 5000 ;(\mathrm{e}), \times 8000 ;(\mathrm{f}), \times 10000$
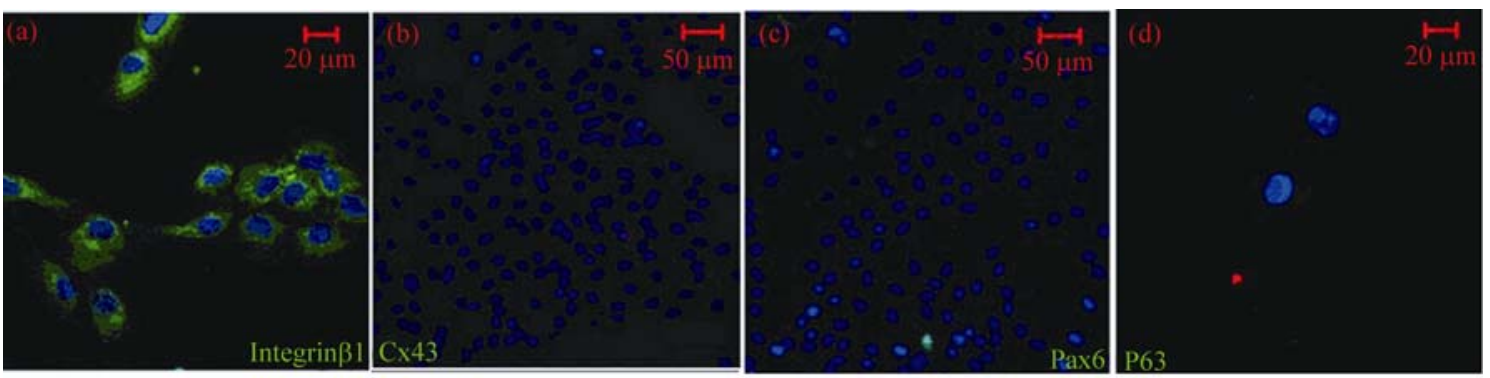

图 6 诱导前 MSC integrin $\beta 1(a) 、 C x 43(b) 、 P a x 6(c)$ 和 P63(d)的免疫苂光检测 

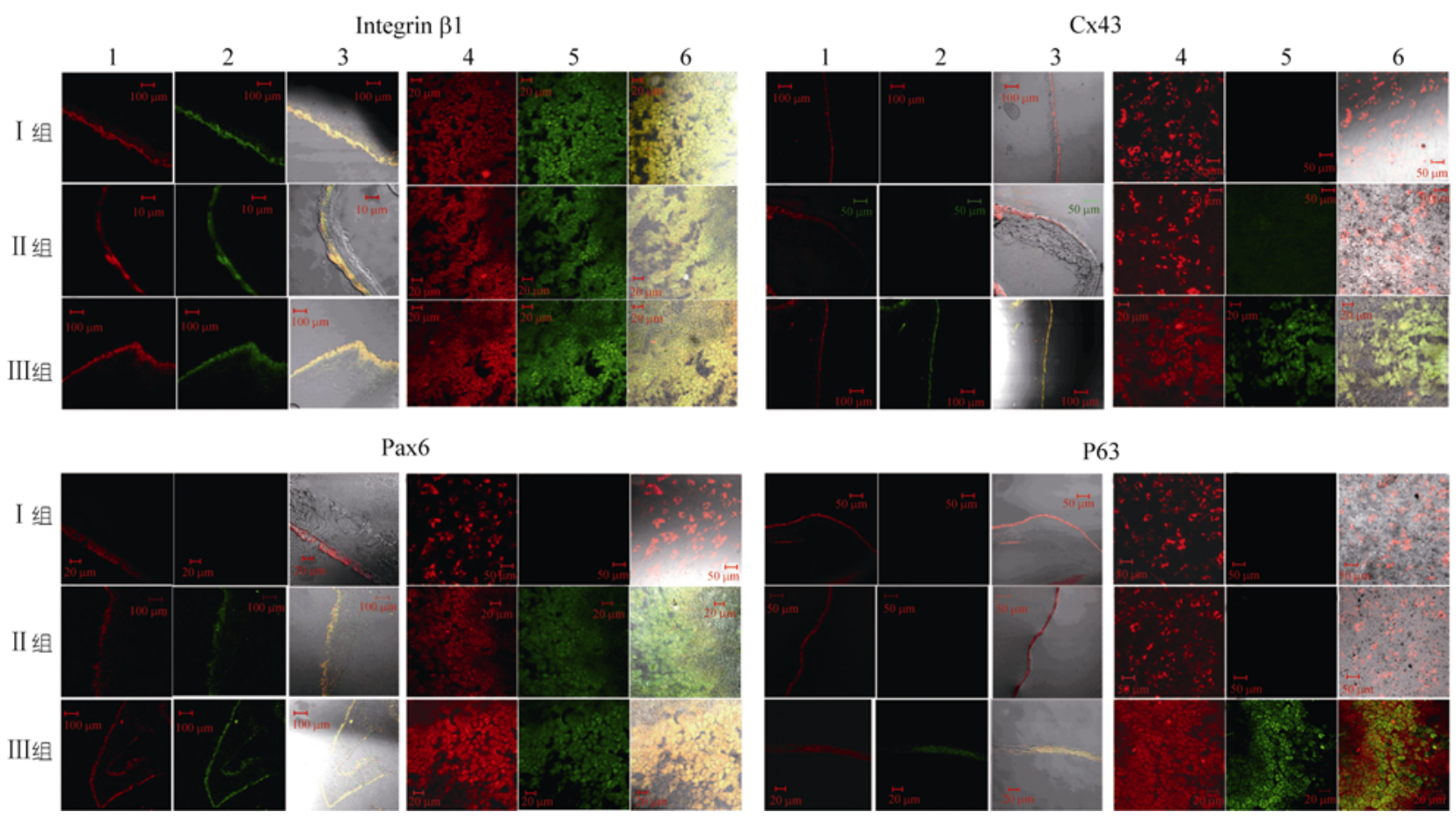

P63

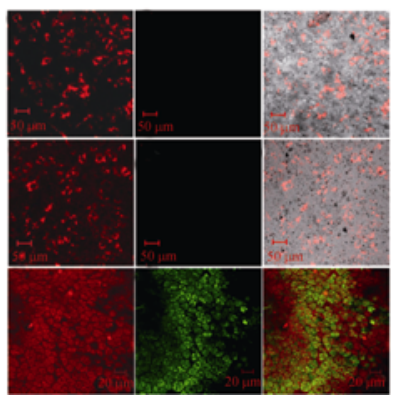

图 7 诱导 $10 \mathrm{~d}$ 后 I 组、 II 和 III 组 MSC 的纵切面观(1 3)和横切面观(4 6)

1 和 4, cM-DiI 标记的 MSC; 2 和 5, MSC 标记蛋白染色; 3 和 6, MSC 的 cM-DiI 和蛋白双标记
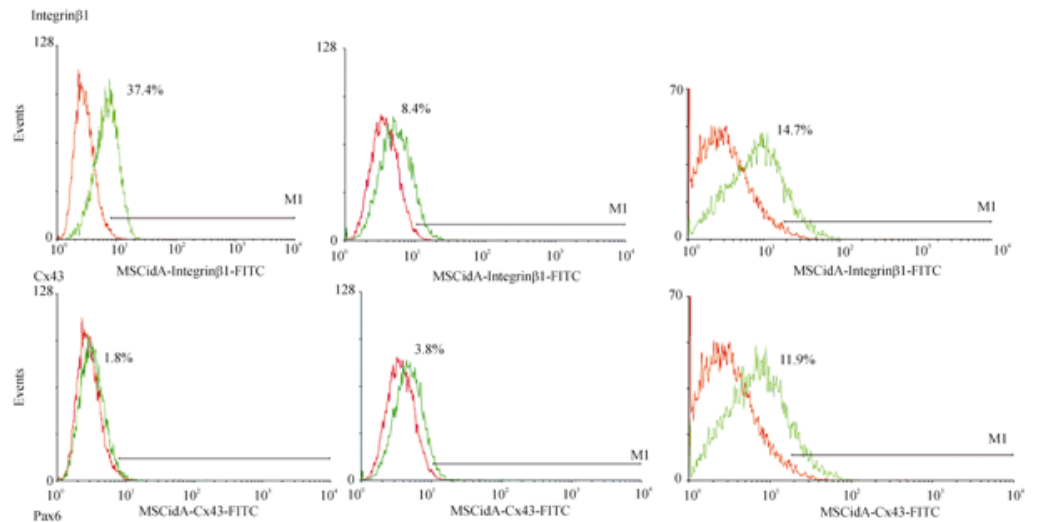

\begin{tabular}{cccc}
\hline 活细胞百分数 $(\%)$ & I 组 & II 组 & III组 \\
\hline Integrin $\beta 1$ & $41.99 \pm 5.04$ & $10.68 \pm 2.05$ & $16.77 \pm 1.89$ \\
Cx43 & $1.82 \pm 0.11$ & $3.78 \pm 0.36$ & $11.89 \pm 0.62$ \\
Pax6 & $1.41 \pm 0.12$ & $6.34 \pm 0.48$ & $12.87 \pm 0.37$ \\
P63 & $0.98 \pm 0.18$ & $1.92 \pm 0.49$ & $7.89 \pm 1.33$ \\
\hline
\end{tabular}
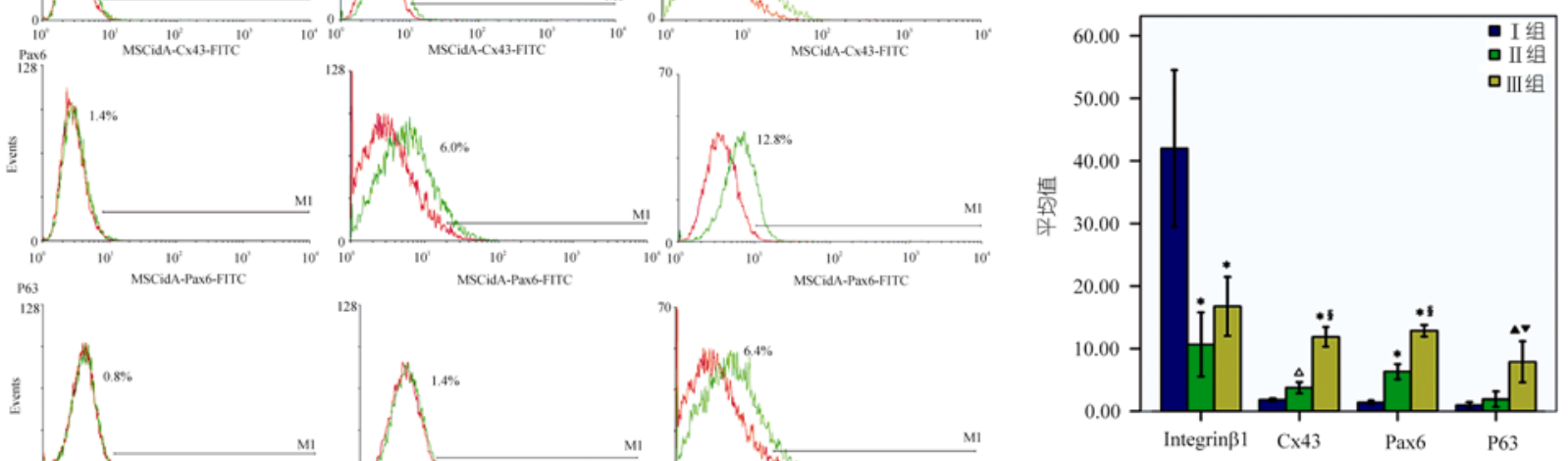

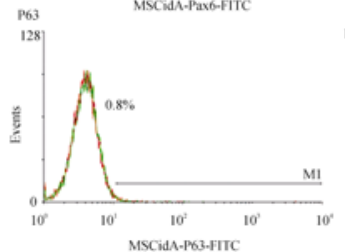

(a)

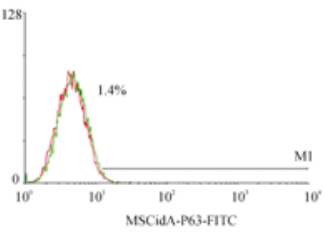

(b)

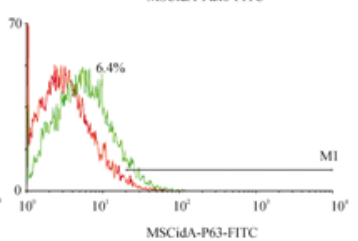

(c)

图 8 流式细胞仪检测 I (a)、 II (b)和 III 组(c)细胞表达 integrin $\beta 1, C x 43$, Pax6 和 P63 的流式图

红色显示阴性对照. 直方图显示各组阳性细胞数统计学差异(d). * $P<0.001$, 与 I 组比较; $\triangle, P<0.01$, 与 I 组比较; $\S, P<0.001$, 与 II 组比较;

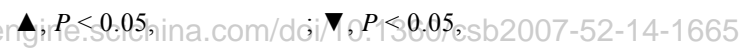


分化被认为违背了发育过程中的生物学常识. 然而, 近几年的研究显示, MSC在特定的情况下能够表现出 其他胚层组织细胞特有的表达谱和功能表型 [29 31], 并能表达 3 个胚层细胞的mRNA转录特点 ${ }^{[32]}$, 体现了 干细胞的多向性. 目前, 关于MSC分化为 $E C$ 的研究 报道甚少. 本研究通过一种新的体外共孵育体系, 研 究了MSC向EC分化.

MSC 作为细胞治疗具有获取方便、易分离培养 和扩增等优势. 然而, 在体外扩增培养过程中, 由于 细胞生存环境的改变有可能影响其生理特性. 为了 尽可能避免传代过程对细胞的影响, 本研究选用 P3 代细胞, 并经多向分化实验证实细胞能成功分化为 骨细胞和脂肪细胞, 确定所用的细胞符合 MSC 特征.

MSC 标记是进行分化实验的一个重要步骤。 cM-DiI 是一种发橘红色菼光的亲脂性膜染料, 能很 好地长期示踪细胞. 本研究证实, cM-DiI 标记后苂光 在胞内表达稳定, 阳性标记率达 $98 \%$ 以上, 标记细胞 形态良好, 能有效地观察细胞在体外的诱导分化情 况.

羊膜是一种天然的高分子生物材料, 含有与角 膜基质相似的胶原、糖蛋白、蛋白多糖、整合素等多 种成分, 能为细胞的增殖、分化提供丰富的营养成分 [33,34]. 羊膜经去上皮细胞处理后, 既能消除上皮细胞 成分的免疫源型, 又能增强细胞綮附及促进细胞分 化，是体外诱导细胞分化的良好载体.

关于 $\mathrm{MSC}$ 在体外向 $\mathrm{EC}$ 分化的研究, 关键问题是 如何创造符合体内环境的诱导条件及如何鉴定其向 $\mathrm{EC}$ 分化, 对此我们在总结预实验的基础上, 设计了 一种体外共孵育体系, 这种体系允许MSC与EC进行 信息交流而避免直接接触. 采用不同条件诱导, 发现 用基础培养液培养时出现与 MSC可塑性的阴性报道 类似的结果. 当加入一类与调节细胞增殖、分化密切 相关 ${ }^{[35 ~ 39]}$ 的生长因子培养时, 细胞形态虽无明显改 变, 但标志蛋白表达改变, 显示出一定的转分化效 果, 推测这种有限的诱导能力可能与培养环境中生 长因子的量或细胞种属来源有关. 当添加LPS时, MSC发生较明显的表型转变. LPS 是重要的致炎因 子, 能介导实质细胞激活, 诱导多种细胞因子、生长 因子等的合成和释放, 引起细胞生物学特性改变, 这 种作用通过细胞间和细胞-细胞外基质的相互作用而 影响干细胞的增殖、分化及迁移 ‘40,41].
在标记物选择方面, $\mathrm{Cx} 43$ 表达于成熟角膜上皮 细胞及角膜 $\mathrm{TAC}$ 细胞 ${ }^{[42]} ; \mathrm{P} 63$ 在角膜缘基底细胞表达 [43]. 诱导后的MSC表达 $\mathrm{Cx} 43$ 和P 63 , 提示细胞在适宜 的条件下能分化为不成熟的角膜上皮样细胞. Pax6 是一种转录调控因子, 在成体角膜上皮细胞、角膜缘 干细胞广泛表达, 对角膜上皮组织发育和角膜上皮 细胞表型维持起到关键作用 ${ }^{[44]}$, 并能有效启动细胞 黏附蛋白和角蛋白 $\mathrm{K} 12$ 的转录 ${ }^{[45]}$. 诱导细胞Pax 6 的 表达上调提示Pax6 可能通过调节MSC与EC及羊膜的 相互作用而促进其转分化过程. Integrin $\beta 1$ 是一组介 导细胞间和细胞-细胞外基质间黏附的跨膜蛋白, 其 表达丧失或改变提示干细胞的分化或凋亡 ${ }^{[46,47]}$. 诱 导细胞integrin $\beta 1$ 表达下调, 推测细胞可能通过调控 细胞间或细胞-细胞外基质的结合而调控细胞的分化 命运. 总之, 本研究结果显示, MSC在适宜的条件下 可能分化为接近角膜上皮前体细胞性质的细胞群, 这一结果与体内组织损伤后干细胞通过分化依赖机 制进行组织修复的生理现象相符 ${ }^{[48]}$. 但MSCs的诱导 分化是一个复杂的过程, 多种因素包括年龄都可能 影响分化结果. 进一步的实验需要针对不同年龄层 次的MSC进行诱导实验, 并对分化后细胞表型的维 持及分化机制等问题进行深入探讨.

另外, 研究中还发现, 诱导中的 MSC 较诱导前 增殖缓慢, 推测可能与细胞的旁分泌有关. 细胞间及 细胞-细胞外基质的相互作用可促进细胞合成和分泌 多种生长因子和细胞因子, 这些因子又通过调节细 胞间与细胞-细胞外基质的相互作用而抑制干细胞增 殖、促进分化. 关于何种信号转导途径参与了这种增 殖与分化的调控, 目前正在研究中.

总之, 本研究结果显示, 在适宜条件下, 灵长类 动物恒河猴 MSC 能够诱导分化为角膜上皮前体样细 胞, 有可能作为构建生物角膜的种子细胞来源, 进行 眼表修复. MSC 由于其取材容易、在体外易分离和扩 增培养, 在长期培养过程中, 始终保持其多向分化潜 能, 遗传背景稳定, 自体移植可避免免疫排斥反应, 是组织工程学和创伤愈合研究中的理想种子细胞, 在眼组织工程中具有广泛的应用前景.

\section{参考文献}

1 Morgan S, Murray A. Limbal autotransplantation in the acute and chronic phases of severe chemical injuries. Eye, 1996, 10: 349354

2 Pellegrini G, Traverso C E, Franzi A T, et al. Long-term restoration of damaged corneal surfaces with autologous cultivated corneal 
epithelium. Lancet, 1997, 49: 990-993 [DOI]

3 Tsubota K, Satake Y, Kaido M, et al. Treatment of severe ocular-surface disorders with corneal epithelial stem-cell transplantation. N Engl J Med, 1999, 340: 1697-1703 [DOI]

4 Holland E J, Schwartz G S. Epithelial stem cell transplantation for severe ocular surface disease. N Engl J Med, 1999, 340: 17521753 [DOI]

5 Tsai R J, Li L M, Chen J K. Reconstruction of damaged corneas by transplantation of autologous limbal epithelial cells. N Engl J Med, 2000, 343: 86-93 [DOI]

6 Li Q J, Ashraf F M, Rana T S, et al. Long-term survival of allogeneic donor cell-derived corneal epithelium in limbal deficient rabbits. Curr Eye Res, 2001, 23: 336-345 [DOI]

7 Solomon A, Ellies P, Anderson D F, et al. Long-term outcome of keratolimbal allograft with or without penetrating keratoplasty for total limbal stem cell deficiency. Ophthalmology, 2002, 109: 1159 -1166 [DOI]

8 Reinhard T, Spelsberg H, Henke L, et al. Long-term results of allogeneic penetrating limbo-keratoplasty in total limbal stem cell deficiency. Ophthalmology, 2004, 111: 775-782 [DOI]

9 Sharpe J R, Daya S M, Dimitriadi M, et al. Survival of cultured allogeneic limbal epithelial cells following corneal repair. Tissue Eng, 2007, 13: 123-132 [DOI]

10 Nakamura T, Endo K, Cooper L J, et al. The successful culture and autologous transplantation of rabbit oral mucosal epithelial cells on amniotic membrane. Invest Ophthalmol Vis Sci, 2003, 44: 106116 [DOI]

11 Lumelsky N, Blondel O, Laeng P, et al. Differentiation of embryonic stem cells to insulin-secreting structures similar to pancreatic islets. Science, 2001, 292: 1389-1394 [DOI]

12 Zhang S C, Wernig M, Duncan I D, et al. In vitro differentiation of transplantable neural precursors from human embryonic stem cells. Nat Biotechnol, 2001, 19: 1129-1133 [DOI]

13 Chinzei R, Tanaka Y, Shimizu-Saito K, et al. Embryoid-body cells derived from a mouse embryonic stem cell line show differentiation into functional hepatocytes. Hepatology, 2002, 36: 22-29 [DOI]

14 Ali N N, Edgar A J, Samadikuchaksaraei A, et al. Derivation of type II alveolar epithelial cells from murine embryonic stem cells. Tissue Eng, 2002, 8: 541-550 [DOI]

15 Kawasaki H, Suemori H, Mizuseki K, et al. Generation of dopaminergic neurons and pigmented epithelia from primate ES cells by stromal cell-derived inducing activity. Proc Natl Acad Sci USA, 2002, 99: 1580-1585 [DOI]

16 Chiba S, Iwasaki Y, Sekino H, et al. Transplantation of motoneuron-enriched neural cells derived from mouse embryonic stem cells improves motor function of hemiplegic mice. Cell Transplant, 2003, 12: 457-468

17 Chiba S, Ikeda R, Kurokawa M S, et al. Anatomical and functional recovery by embryonic stem cell-derived neural tissue of a mouse model of brain damage. J Neurol Sci, 2004, 219: 107-117 [DOI]

18 Ye J, Yao K, Kim J C. Mesenchymal stem cell transplantation in a rabbit corneal alkali burn model: Engraftment and involvement in wound healing. Eye, 2006, 20: 482-490 [DOI]

19 郭䑣，王薇，张君，等. 骨髓间充质干细胞移植治疗眼表损害的
初步实验研究. 中华眼科杂志, 2006, 42: 246-250

20 Ma Y, Xu Y, Xiao Z, et al. Reconstruction of chemically burned rat corneal surface by bone marrow-derived human mesenchymal stem cells. Stem Cells, 2006, 24: 315-321 [DOI]

21 Izadpanah R, Joswig T, Tsien F, et al. Characterization of multipotent mesenchymal stem cells from the bone marrow of rhesus macaques. Stem Cells Dev, 2005, 14: 440-451 [DOI]

22 Pittenger M F, Mackay A M, Beck S C, et al. Multilineage potential of adult human mesenchymal stem cells. Science, 1999, 284: $143-147$ [DOI]

23 Horan P K, Melnicoff M J, Jensen B D, et al. Fluorescent cell labeling for in vivo and in vitro cell tracking. Methods Cell Biol, 1990, 33: 469-490

24 Fukuhara S, Tomita S, Nakatani T, et al. Comparison of cell labeling procedures for bone marrow cell transplantation to treat heart failure: Long-term quantitative analysis. Transplant Proc, 2002, 34: $2718-2721$ [DOI]

25 Liu J, Song G, Wang Z. Establishment of a corneal epithelial cell line spontaneously derived from human limbal cells. Exp Eye Res, 2007, 84: 599-609 [DOI]

26 Rama P, Giannini R, Bruni A, et al. Further evaluation of amniotic membrane banking for transplantation in ocular surface diseases. Cell Tissue Bank, 2001, 2: 155-163 [DOI]

27 Kruse F E, Joussen A M, Rohrschneider K, et al. Cryopreserved human amniotic membrane for ocular surface reconstruction. Graefes Arch Clin Exp Ophthalmol, 2000, 238: 68-75 [DOI]

28 Friedenstein A J, Gorskaja U, Kalugina N N. Fibroblast precursors in normal and irradiated mouse hematopoietic organs. Exp Hematol, 1976, 4: 267-274

29 Woodbury D, Schwarz E J, Prockop D J, et al. Adult rat and human bone marrow stromal cells differentiate into neurons. J Neurosci Res, 2000, 61: 364-370 [DOI]

30 Liechty K W, MacKenzie T C, Shaaban A F, et al. Human mesenchymal stem cells engraft and demonstrate site-specific differentiation after in utero transplantation in sheep. Nat Med, 2000, 6: 1282 -1286 [DOI]

31 Krause D S.Engraftment of bone marrow-derived epithelial cells. Ann N Y Acad Sci, 2005, 1044: 117-124 [DOI]

32 Tremain N, Korkko J, Ibberson D, et al. MicroSAGE analysis of 2,353 expressed genes in a single cell-derived colony of undifferentiated human mesenchymal stem cells reveals mRNAs of multiple cell lineages. Stem cells, 2001, 19: 408—418 [DOI]

33 Modesti A, Scarpa S, D’Orazi G, et al. Localization of type IV and $\mathrm{V}$ collagens in the stroma of human amnion. Prog Clin Biol Res, 1989, 296: 459-463

34 Fukuda K, Chikama T, Nakamura M, et al. Differential distribution of subchains of the basement membrane components type IV collagen and laminin among the amniotic membrane, cornea and conjunctiva. Cornea, 1999, 18: 73-79 [DOI]

35 DiRenzo J, Signoretti S, Nakamura N, et al. Growth factor requirements and basal phenotype of an immortalized mammary epithelial cell line. Cancer Res, 2002, 62: 89-98

36 Vicario-Abejon C, Yusta-Boyo M J, Fernandez-Moreno C, et al. Locally born olfactory bulb stem cells proliferate in response to insulin-related factors and require endogenous insulin-like growth 
factor- 1 for differentiation into neurons and glia. J Neurosci, 2003, 23: 895-906

37 Harris R C. Potential physiologic roles for epidermal growth factor in the kidney. Am J Kidney Dis, 1991, 17: 627-630

38 Wolfgang C L, Lin C, Meng Q, et al. Epidermal growth factor ac- tivation of intestinal glutamine transport is mediated by mitogen-activated protein kinases. J Gastrointest Surg, 2003, 7: 149-156 [DOI]

39 Rubin A L, Rice R H. Differential regulation by retinoic acid and calcium of transglutaminases in cultured neoplastic and normal human keratinocytes. Cancer Res, 1986, 46: 2356-2361

40 Chang J, Zhang C, Tani-Ishii N, et al. NF-kappaB activation in human dental pulp stem cells by TNF and LPS. J Dent Res, 2005, 84: 994-998

41 Abdallah B M, Boissy P, Tan Q, et al. DLK1/FA1 regulates the function of human bone marrow mesenchymal stem cells (HMSC) by modulating gene expression of pro-inflammatory cytokines and immune-response-related factors. J Biol Chem, 2007, 282: 73397351; Epub 2006 Dec 19 [DOI]

42 Matic M, Petrov I N, Chen S, et al. Stem cells of the corneal epithelium lack connexins and metabolite transfer capacity. Differentiation, 1997, 61: 251-260 [DOI]
43 Pellegrini G, Dellambra E, Golisano O, et al. p63 identifies keratinocyte stem cells. Proc Natl Acad Sci USA, 2001, 98: 3156-3161 [DOI]

44 Sivak J M, Mohan R, Rinehart W B, et al. Pax-6 expression and activity are induced in the reepithelializing cornea and control activity of the transcriptional promoter for matrix metalloproteinase gelatinase B. Dev Biol, 2000, 222: 41-54 [DOI]

45 Wolosin J M, Budak M T, Akinci M A.Ocular surface epithelial and stem cell development. Int J Dev Biol, 2004, 48: 981-991 [DOI]

46 Heckmann L, Fiedler J, Mattes T, et al. Mesenchymal progenitor cells communicate via alpha and beta integrins with a three-dimensional collagen type I matrix. Cells Tissues Organs, 2006, 182: $143-154$ [DOI]

47 Nakagawa H, Akita S, Fukui M, et al. Human mesenchymal stem cells successfully improve skin-substitute wound healing. Br J Dermatol, 2005, 153(1): 29-36 [DOI]

48 Wagers A J, Sherwood R I, Christensen J L, et al. Little evidence for developmental plasticity of adult hematopoietic stem cells. Science, 2002, 297: 2256-2259 [DOI] 